\title{
UNA ESPECIE NUEVA DEL GÉNERO NOPS MACLEAY, 1839 \\ (ARANEAE, CAPONIIDAE) PROCEDENTE DE ISLAS VÍRGENES DE ESTADOS UNIDOS DE AMÉRICA, ANTILLAS MENORES
}

\author{
Alexander Sánchez-Ruiz \\ Centro Oriental de Ecosistemas y Biodiversidad, Museo de Historia Natural "Tomás Romay". José A. Saco \\ \# 601, Santiago de Cuba 90100, Cuba. alex@bioeco.ciges.inf.cu
}

\section{RESUMEN}

Se describe una nueva especie de araña del género Nops MacLeay, 1839, procedente de Little St. James, Islas Vírgenes de Estados Unidos de América, Antillas Menores. Con este hallazgo se eleva a 16 especies la fauna de Caponiidae de las Antillas. Se incluye además una lista anotada de las 13 especies de arañas del género Nops presentes en las Antillas y se discuten aspectos de su distribución.

Palabras clave: Araneae, Caponiidae, Nops, nueva especie, Islas Vírgenes.

\section{ABSTRACT}

A new species of the spider genus Nops MacLeay, 1839 is described from Little St. James, United States Virgin Islands, Lesser Antilles. Finding this new species, the Caponiidae fauna of the West Indies is raised to 16 species. An annotated check list of the 13 spider species of the genus Nops from the West Indies is included and some aspects about their distribution are discussed.

Key words: Araneae, Caponiidae, Nops, new species, Virgin Islands.

\section{INTRODUCCIÓN}

La fauna de arañas de las Islas Vírgenes de los Estados Unidos de América todavía no ha sido lo suficientemente estudiada. Entre los primeros y más importantes estudios están los de Petrunkevitch (1926) y Bryant (1942), quienes describen y registran la mayor parte de las arañas de estas islas. Posteriormente la riqueza se va incrementando a partir de las descripciones y registros de especies en obras de revisiones a nivel genérico, fundamentalmente de las arañas de América Central y Las Antillas. En este sentido se destacaron Chickering (1967a, 1967b, 1968a, 1968b, 1968c, 1969, 1971, 1973), Exline y Levi (1962), Archer (1963), Platnick (1975, 1978), Platnick y Shadab (1976, 1977, 1979, 1980, 1982, 1989). Finalmente, Muchmore (1993) publica una lista de los invertebrados terrestres de St. John incluyendo varios nuevos registros de arañas.

La familia Caponiidae está representada en estas islas únicamente por Nops blandus (Bryant, 1942), descrita originalmente a partir de un espécimen macho procedente de Christiansted en St. Croix y colectado por Harry A. Beatty. En la descripción original, Bryant (1942) la ubica bajo el género Caponina Simon, 1891 y posteriormente es transferida por Chickering (1967a) al género Nops MacLeay, 1839. Además de la localidad tipo, Chickering (1967a) la registra de St. John y asigna con dudas a esta especie otros especímenes de Saint Thomas y de Virgin Gorda (Islas Vírgenes Británicas). Esta especie es uno de los Nops antillanos menos restringidos geográficamente, se conoce además de dos localidades en La Hispaniola: Puerto Príncipe, Haití (Bryant, 1948 como Nops coccineus) y Puerto Escondido, provincia Independencia, República Dominicana (Sánchez-Ruiz, 2005a).

Durante la revisión de la colección de Caponiidae en el Museo Americano de Historia Natural, $(\mathrm{AMNH})$, Nueva York, EE. UU., fue encontrado un espécimen macho, perteneciente al género Nops, dentro de un pequeño frasco en un recipiente que contenía varias arañas procedentes de Islas Vírgenes de Estados Unidos. El macho en cuestión fue colectado en Little St. James, una pequeña isla al Sur de Saint Thomas, y la forma del émbolo en los pedipalpos no se corresponde con ninguna de las especies conocidas del género, por lo cual es descrito como una nueva especie en este trabajo. 


\title{
MATERIALES Y MÉTODOS
}

Las mediciones fueron hechas utilizando un estereomicroscopio Carl Zeiss Stemi 2000-C, equipado con un micrómetro de escala lineal. Todas las medidas están expresadas en milímetros. Las mediciones de las patas fueron hechas siguiendo a Galiano (1962) con las modificaciones de Alayón (1976), el largo total de las patas corresponde a la suma de las longitudes de cada artejo medido. La descripción de la coloración se realizó a partir del espécimen en alcohol etílico y bajo una luz blanca procedente de una lámpara de fibra óptica. Para el estudio de los órganos genitales masculinos, los pedipalpos fueron separados del cuerpo del macho para facilitar su orientación e ilustración. Para su conservación los pedipalpos fueron guardados en pequeños frascos de vidrio con alcohol etílico 75\%, junto al espécimen al cual pertenecían. Los dibujos se basaron en fotografías impresas tomadas con una cámara digital Canon Power Shot A620 de 7.1 megapíxeles acoplada al estereomicroscopio, posteriormente se acentuó con tinta china utilizando diferentes puntos de dibujo. Las ilustraciones fueron digitalizadas y corregidas en ordenador usando el software Adobe PhotoShop CS2 ver.9.0.

Se siguió un orden alfabético para ordenar las especies de la lista anotada. Los nombres de los países en mayúsculas sostenidas, Haití y República Dominicana representados por LA HISPANIOLA. Entre paréntesis se destacan la referencia del registro de literatura o la abreviatura de la colección aracnológica donde se examinaron especímenes: Centro Oriental de Ecosistemas y Biodiversidad, Santiago de Cuba, Cuba (BSC.AR); Museo Americano de Historia Natural, $(\mathrm{AMNH})$, Nueva York, EE. UU. (AMNH); Museo de Zoología Comparada, Massachusetts, EE. UU. (MCZ); Colección personal de Giraldo Alayón García, San A. de los Baños, La Habana, Cuba (CGA). Todo el material adicional examinado constituye nuevos registros. Con un asterisco está señalada la localidad tipo de cada especie.

\author{
RESULTADOS \\ SISTEMÁTICA \\ Familia Caponiidae \\ Género Nops MacLeay, 1839 \\ Nops virginicus sp nov. \\ (Tabla 1, Figs. 1 y 2)
}

Diagnosis: Palpo del macho de Nops virginicus sp. nov. con un bulbo globoso, émbolo corto y cónico con la terminación formando una punta (Fig. 1). Se diferencia del único Caponiidae registrado de Islas Vírgenes: $N$. blandus (Bryant, 1942) por la forma del émbolo y por tener el émbolo más corto que el largo del bulbo. La forma del émbolo de esta especie la distingue del resto de los Nops antillanos con émbolos cortos (N. coccineus Simon, 1891; N. enae Sánchez-Ruiz, 2004; N. flutillus Chickering, 1967; N. gertschi Chickering, 1967; N. siboney Sánchez-Ruiz, 2004; N. toballus Chickering, 1967). Las hembras son desconocidas.

Diagnosis: Male palpi of $N$. virginicus sp. n. with a globose bulb, short and conical embolus with termination forming a tip (Fig. 1). Differs of the only Caponiidae recorded from Virgin Islands: $N$. blandus (Bryant, 1942) by the form of the embolus and by having the embolus shorter than the long of the bulb. The form of the embolus of this species distinguishes it from the rest of the antillean Nops with short embolus (N. coccineus Simon, 1891; N. enae Sánchez-Ruiz, 2004; N. flutillus Chickering, 1967; N. gertschi Chickering, 1967; N. siboney Sánchez-Ruiz, 2004; N. toballus Chickering, 1967). Females are unknown. 


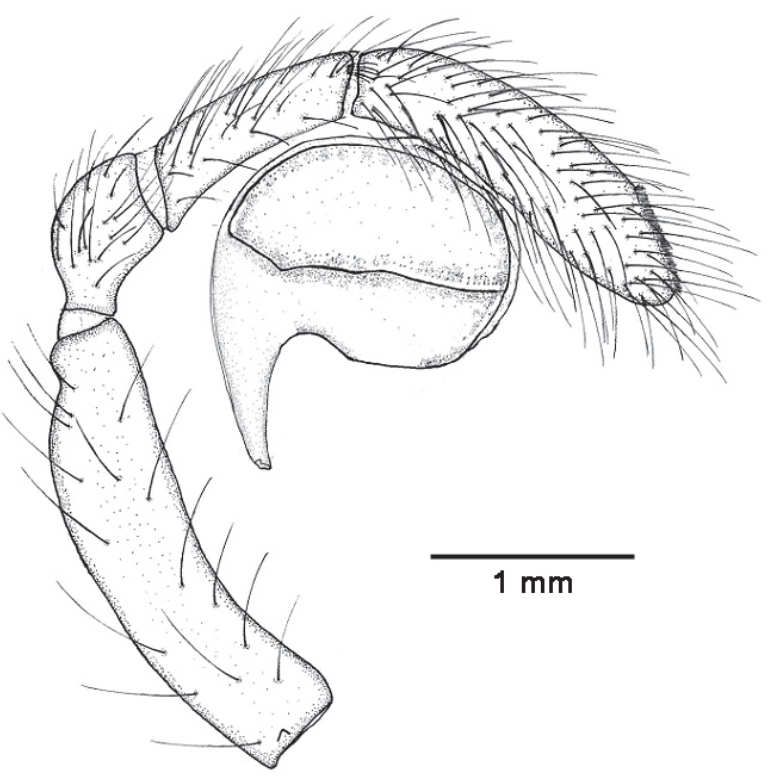

Figura 1. Vista prolateral del palpo izquierdo del macho holotipo de $N$. virginicus sp. nov.

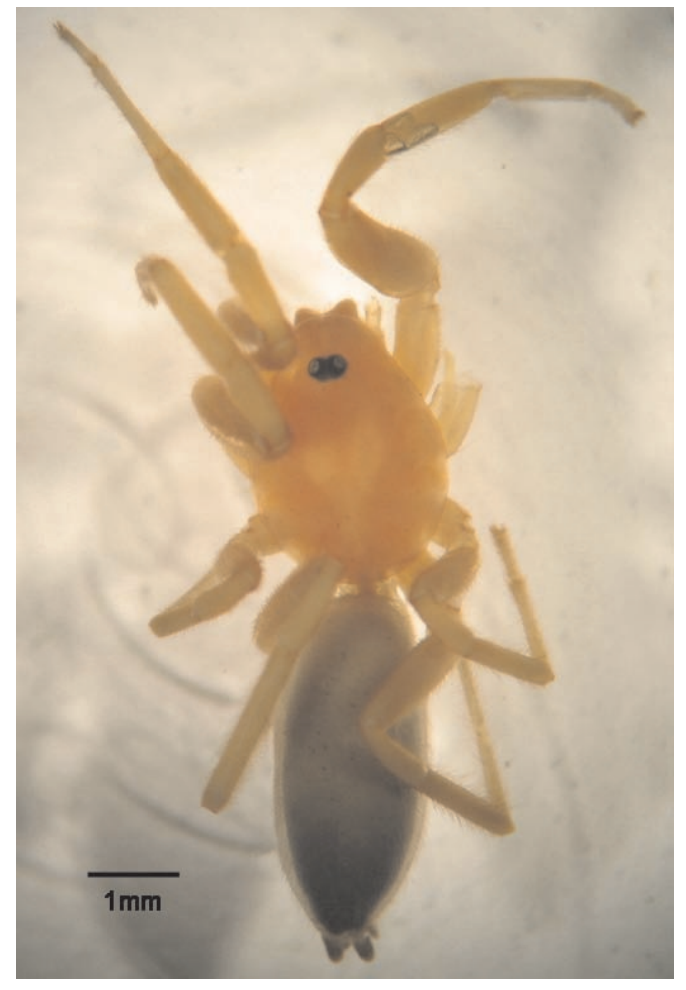

Figura 2. Fotografía del macho holotipo de $N$. virginicus sp. nov.

Descripción del holotipo: Macho. Color en alcohol. El prosoma de color uniforme anaranjado claro con el tubérculo ocular negro. Patas anaranjado claro, pero más claro que el prosoma; quelíceros anaranjado amarillento; pedipalpos anaranjado amarillento. Esternón de igual color que el prosoma pero con una línea anaranjada oscura en todo el margen. Enditos y labio más claros que el esternón. Abdomen dorsalmente gris pálido amarillento y mucho más claro hacia la parte apical; ventralmente de igual coloración pero más claro. Surco genital gris pálido claro, tubérculo anal e hilanderas de un gris más oscuro. Principales caracteres taxonómicos: Carapacho oval ligeramente más estrecho a nivel del área ocular, ancho mayor del carapacho menor que la distancia desde la base del tubérculo ocular a la parte distal del carapacho, redondeado a nivel del clypeus; con dos ojos ovales de diámetro mayor de 0,45 sobre un tubérculo ocular ligeramente elevado y separados una distancia igual al diámetro de estos. Quelíceros con proyección anterior y con una membrana blanca lobulada en el margen interno. Pedipalpos con abundantes pelos, sin uñas, patella corta, tibia con un parche de setas largas prolaterales en la parte distal, cymbium ligeramente expandido, no puntiagudo, con una conspicua y densa mancha de pelos cortos y delgados dorsoapicales y pelos largos ventroapicales; con la tibia del pedipalpo excavada, cymbium con bulbo globoso (0,80 largo y 0,64 ancho), émbolo corto ( 0,34 largo y 0,14 ancho mayor) terminado en punta (Fig. 1). Patas I-II con una quilla traslúcida en los metatarsos y una pequeña extensión ventral de la membrana entre el metatarso y el tarso (0,50 largo extensión I; 0,60 largo extensión II); todos los tarsos subsegmentados 2:2:2:2; uña impar elongada en los tarsos I-II, casi de igual tamaño que las uñas pares; metatarsos enteros; tricobotrias largas en el tarso, metatarso y tibia. Coxas con una proyección submarginal en la base en todas las patas. Esternón oval alargado, más largo que ancho, el ancho mayor a nivel de la coxa II y III; membrana cefalotoráxica sin escleritos epiméricos dorsales; con tres extensiones triangulares del esternón alargadas alcanzando la mitad basal de las coxas II, III y IV. Labio unido al esternón formando una sola pieza, más largo que ancho y terminado en punta. Enditos convergentes, aunque las puntas no se tocan, sin protuberancias posteriores, con la parte media más ancha que la distal y proximal y formado un ángulo obtuso de aproximadamente 120 grados. Hilanderas según la ordenación típica de la familia. Mediciones: prosoma 3,65 largo y 2,60 ancho; esternón 2,30 largo y 1,70 ancho; labio 0,65 largo; abdomen 4,50 largo y 2,25 ancho; largo total del cuerpo 9,15. Las mediciones de las patas y pedipalpo en la Tabla 1. 
Tabla 1. Mediciones (en mm) de las patas y pedipalpo (p) del macho holotipo de Nops virginicus sp. nov.

\begin{tabular}{lccccc}
\hline & I & II & III & IV & $\mathrm{p}$ \\
\hline Coxa & 1,40 & 1,30 & 0,70 & 1,05 & 1,30 \\
Trocanter & 0,45 & 0,45 & 0,35 & 0,75 & 1,15 \\
Fémur & 2,55 & 2,50 & 2,35 & 2,80 & 3,25 \\
Patela & 1,35 & 1,25 & 1,0 & 1,35 & 1,25 \\
Tibia & 1,75 & 1,75 & 1,50 & 2,55 & 1,65 \\
Metatarso & 1,60 & 1,60 & 1,45 & 2,55 & - \\
Tarso & 0,75 & 0,70 & 0,75 & 0,80 & 2,75 \\
\hline TOTAL & 9,85 & 9,55 & 8,10 & 11,85 & 11,35 \\
\hline
\end{tabular}

Etimología. Nombre en aposición, referido a la localidad tipo.

Material tipo. Holotipo $\widehat{\jmath}$ (BSC.AR.656) Islas Vírgenes de Estados Unidos de América: Little St. James, noviembre 13, 1966, Island Project Staff, University of Puerto Rico (sin más datos).

Distribución. Conocida sólo de la localidad tipo en las Islas Vírgenes de Estados Unidos de América.

Comentarios. Especímenes adicionales al holotipo y las hembras referidas a esta especie no han podido ser localizados; aunque el macho holotipo posee características inequívocas que lo separa del resto de las especies conocidas.

\section{LISTA ANOTADA DE LAS ESPECIES DE ARAÑAS DEL GÉNERO NOPS DE LAS ANTILLAS}

\section{N. blandus (Bryant, 1942)}

Registros literatura. ISLAS VÍRGENES DE EE.UU.: St. Croix* (Bryant, 1942); St. John (Chickering, 1967a y Muchmore, 1993); ISLAS VÍRGENES REINO UNIDO: Virgin Gorda (Chickering, 1967a); LA HISPANIOLA: Puerto Principe, Haití (Bryant, 1948 como Nops coccineus); Puerto Escondido, República Dominicana (Sánchez-Ruiz, 2005a).

Material adicional. ISLAS VÍRGENES DE EE.UU.: 10̄ (AMNH), St. John, jul. 1996.

2. N. coccineus Simon, 1891

Registros literatura. SAN VICENTE Y LAS GRANADINAS: Isla de San Vicente* (Simon, 1891).

3.N. craneae Chickering, 1967

Registros de literatura. TRINIDAD Y TOBAGO: Simla*, Arima Valley, Isla de Trinidad (Chickering, 1967a); Balandra Bay, Isla de Trinidad (Chickering, 1967a).

4. N. enae Sánchez-Ruiz, 2004

Registros de literatura. CUBA: Piedra la Vela*, Yateras, Guantánamo (Sánchez-Ruiz, 2004); Universidad de Oriente, Santiago de Cuba (Sánchez-Ruiz, 2004); La Pimienta, Tercer Frente, Santiago de Cuba (Sánchez-Ruiz, 2004).

Material adicional. CUBA: $1 \lesssim, 1$ 우 (BSC.AR 542), La China, Sierra Cristal, Santiago de Cuba, 27 marzo 2001. R. Teruel. 10 (BSC.AR 539), Carretera a Yaguaneque, $1 \mathrm{Km}$. después de bifurcación a Sagua, Moa, Holguín, 26 sept. 2005, R. Teruel. 
1 오 (BSC.AR 552), Jardín Botánico de Cupainucú, Guisa, Granma, CUBA, 18 enero 1994, R. Teruel. 19 (BSC.AR 555), Jardín Botánico de San Juan, Santiago de Cuba, CUBA, 11 febrero 1998, R. Teruel. 1 ( (BSC.AR 559), Playa Blanca, Guama, Santiago de Cuba, CUBA, 27 septiembre 2003, R. Teruel y L. F. Armas. 19 (BSC.AR 561), Casacada de Juan Gonzáles, Guama, Santiago de Cuba, CUBA, 10 junio 2009, R. Teruel. 19 (BSC.AR 566), Presa El Avispero, El Cristo, Santiago de Cuba, CUBA, 8 octubre 1997, A. Sánchez. 1 q (BSC.AR 583), El Oro, Sierra Cristal, Segundo Frente, Santiago de Cuba, CUBA, 24 marzo 2001, R. Teruel. 1 q (BSC.AR 584), Centeno, Pueblo Nuevo, Moa, Holguín, CUBA, 20 octubre $2001, \mathrm{R}$. Teruel.

5. N. ernestoi Sánchez-Ruiz, 2005

Registros de literatura. LA HISPANIOLA: Hotel Montaña*, Jarabacoa, La Vega, República Dominicana (Sánchez-Ruiz, 2005a).

6. N. flutillus Chickering, 1967

Registros de literatura. CURAÇAO: S. Nicolaas, St. Martha* (Chickering, 1967a)

Material adicional. CURAÇAO: 1 inmaduro (MCZ), Fuick, Oostpunt, 26 dic. 1962, H. Levi \& B. de Jong. 1 inmaduro (AMNH), Siberie (no mas datos). 1 inmaduro (MCZ), S de Savonet, Shady ravine, 28 dic. 1962, H. Levi \& B. de Jong. 2 inmaduros (MCZ), 3 km N de Savonet, Shady ravine, 28 dic. 1962, H. Levi \& B. de Jong. 4 inmaduros (MCZ), Willemstad, a los lados de la carretera, 24 dic. 1962, H. Levi \& B. de Jong. 2 inmaduros (MCZ), S de Slope Veeris Berg, 20 dic. 1962, H. Levi \& B. de Jong.

\section{N. gertschi Chickering, 1967}

Registros de literatura. LA HISPANIOLA: Loma Cibao*, La Vega, República Dominicana (Chickering, 1967a); Carretera entre Oviedo y Laguna de Oviedo, Pedernales, República Dominicana (Sánchez-Ruiz, 2005a).

Material adicional. LA HISPANIOLA: 2 (CGA), Parque Nacional Jaragua, El Cajuil, Oviedo, provincia Pedernales, República Dominicana, 19 mayo 1999, I. Arias y H. Andújar. 19 (BSC.AR 616), La Charca, Carretera Aceitillar km 16, Sierra de Bahoruco, provincia Pedernales, República Dominicana, 22 junio 2004, A. Sánchez. 1 (BSC.AR 598), Carretera Aceitillar km 7, Sierra de Bahoruco, provincia Pedernales, República Dominicana, 1 febrero 2005, A. Sánchez. 1 q (BSC.AR 516), Carretera Aceitillar km 10, Sierra de Bahoruco, provincia Pedernales, República Dominicana, 20 junio 2006, A. Sánchez.

\section{N. glaucus Hasselt, 1887}

Registros de literatura. BONAIRE: Isla de Bonaire*, Antillas Holandesas (Hasselt, 1887); VENEZUELA: Venezuela (sin más datos) (Simon, 1892).

9. N. guanabacoae MacLeay, 1839

Registros de literatura. CUBA: Guanabacoa*, La Habana (MacLeay, 1839); San A. de los Baños, La Habana (Alayón García, 1977); Santiago de las Vegas, La Habana (Alayón García, 1977); Puente Bacunayagua, Matanzas (Alayón García, 1977); Jardín Botánico de Soledad, Cienfuegos (Alayón García, 1977); Loma de la Guanábana, Isla de la Juventud (Alayón García, 1977); Yaguajay, Minas, Camagüey (Alayón García, 1977); Los Negros, Contramaestre, Santiago de Cuba (Alayón García, 1977); Puerto Boniato, Santiago de Cuba (Alayón García, 1977); Río la Mula, Guama, Santiago de Cuba (Sánchez-Ruiz, 2004); Aguas Claras, Holguín (Teruel \& Sánchez-Ruiz, 2000). 
Material adicional. CUBA: 2ð, 4 ㅇ (BSC.AR 531), Presa Ochoa, Santa Clara, mayo 26, 2006, R. Teruel. $1{ }^{\lambda}$ (BSC.AR 535), El Retiro, Reserva Baconao, Santiago de Cuba, 4 mayo 2006, R. Teruel. $1 \delta^{\lambda}$ (BSC.AR 536), 1 Km. Norte de Río La Mula, Guamá, Santiago de Cuba, 20 junio 2005, A. Sánchez. 1 q (BSC.AR 532), Los Morones, Guama, Santiago de Cuba, junio 23, 1999, A. Sánchez. 2 ( Holguín, sept. 27, 2005, R. Teruel. 1 q (BSC.AR 551), La Cantera, Mella, Santiago de Cuba, 30 mayo 2005, B. Lauranzon. 1 + (BSC.AR 534), Cayo Conuco, Caibarien, Villa Clara, 20 febrero 2006. T. M. Rodríguez. 1 ( (BSC.AR 524), Baconao, Santiago de Cuba, 9 agosto 2008, D. Ortiz. 1 ㅇ (BSC.AR 519), Las Guiras, Santa Clara, Villa Clara, 18 Diciembre 2007, N. Gómez. 19 (BSC.AR 523), Cueva de las Perlas, Península de Guanacahabibes, Pinar del Río. 18 mayo 1998, A. Fong. 3 đ, 9 (BSC.AR 527), El Copey, Placetas, Villa Clara, 1 junio 2009, R. Teruel, T. M. Rodríguez.

10. N. siboney Sánchez-Ruiz, 2004

Registros de literatura. CUBA: $1 \mathrm{Km}$. O de Playa Siboney*, Reserva Siboney-Juticí, Santiago de Cuba (Sánchez-Ruiz, 2004); Boca de Cabañas, Santiago de Cuba (SánchezRuiz, 2004); Cajobabo, Imías, Guantánamo (Sánchez-Ruiz, 2005b).

Material adicional. CUBA: 3 + (BSC.AR 506), Márgenes de Laguna Baconao, Santiago de Cuba, 7 mayo 2001, R. Teruel.

11. N. simla Chickering, 1967

Registros de literatura. TRINIDAD Y TOBAGO: Simla*, Arima Valley, Isla de Trinidad (Chickering, 1967a); Piarco, Isla de Trinidad (Chickering, 1967a). PANAMÁ: Panamá (Nentwig, 1993).

\section{N. toballus Chickering, 1967}

Registros de literatura. JAMAICA: St. Catherine Parish*, cerca de May Pen (Chickering, 1967a); Claredon Parish, 4.8 Km N de May Pen (Chickering, 1967a); Blue Mountains, Main Range (Chickering, 1967a); St. Thomas Parish, Morant Bay (Chickering, 1967a).

Material adicional. JAMAICA: $1 \widehat{\jmath}$ (MCZ), St. Thomas Parish, 14 min. E de Kingston, 25 mayo 1956; 1 inmaduro (MCZ), St. Andrews, parte NW de Long Mountains, 8 mayo 1956; 2 inmaduros (MCZ), St. Thomas, Morant Bay, 4 octubre 1957; 1 ㅇ (MCZ), St. Catherine, Innswood Estate, 10 noviembre 1963; 1 q (MCZ), St. Catherine, Port Henderson, 4 mayo 1956.

13. N. virginicus sp. nov.

ISLAS VÍRGENES DE EE.UU.: Little St. James*, Sur de St. Thomas

\section{DISCUSIÓN}

Con la descripción de un nuevo Nops se incrementa a 13 el número de especies de este género en Las Antillas. No se incluyen en la lista las especies Nops ludovicorum Alayón, 1976 y Nops ariguanabo Alayón, 1986 descritas de Cuba, pues luego de examinar el material tipo de ambas especies, estas no presentan los caracteres diagnósticos del género Nops.

El endemismo en el género Nops es muy alto, de las 26 especies actuales conocidas, solo cinco no son endémicas de sus lugares de origen. En las Antillas, este género presenta una notable especiación y con excepción de N. blandus (Bryant, 1942), N. glaucus Hasselt, 1887 y N. simla Chickering, 1967, el resto de las especies antillanas se conocen de una sola isla. La especie con un mayor ámbito geográfico es Nops blandus, la cual presenta las áreas de distribución más alejadas (Fig. 3) y posiblemente se encuentre en otras áreas intermedias. 
Como era de esperar Cuba y La Hispaniola son las islas con mayor número de especies (4 y 3 respectivamente), ya que presentan la mayor extensión de territorio y la mayor complejidad de hábitats en todo el Caribe insular. En Cuba N. guanabacoae MacLeay, 1839 es la especie con mayor número de registros y aparentemente se encuentra distribuida en todo el archipiélago. Sin embargo, muchos de los registros actualmente asignados a esta especie se basaron sólo en especímenes hembras y mientras no se conozcan caracteres diagnósticos confiables para el sexo, es muy difícil una determinación precisa, pues hasta el momento la mayoría de las hembras de las especies antillanas son muy similares (Sánchez-Ruiz 2005a). Posiblemente, estos registros de $N$. guanabacoae sean realmente un grupo de especies muy afines entre sí (G. Alayón com. pers.). Por otro lado, la forma del émbolo y el bulbo en los machos resultan caracteres diagnósticos confiables para separar las especies de Nops (Chickering, 1967a).

La Región Oriental de Cuba y La Hispaniola, a pesar de haber estado unidas por más tiempo durante el Oligoceno, son las áreas de mayor especiación en el Caribe (Fig. 3). Ambas áreas poseen tres especies, las cuales en algunos de los casos viven simpátricamente. Estos territorios se caracterizan por la presencia de los sistemas montañosos más grandes del Caribe Insular, incluyendo las mayores alturas sobre el nivel del mar, lo que evidentemente ha servido de barrera geográfica para inducir la especiación en este grupo de arañas, adaptadas a vivir preferentemente en ambientes de poca humedad. N. siboney Sánchez-Ruiz, 2004, se distribuye en toda la costa sur oriental de Cuba, desde Santiago de Cuba hasta Guantánamo, solamente bajo piedras y plantas de Agave sp. en el Matorral Xeromorfo Costero y Subcostero. N. enae Sánchez-Ruiz, 2004, ocupa áreas más alejadas de la costa y menos secas, preferentemente en los Bosques Semideciduos hacia el norte viviendo simpátricamente con N. guanabacoae MacLeay, 1839, aunque llega incluso a alturas sobre los $800 \mathrm{msnm}$ en los Bosques de Pinos bajo la corteza de los árboles, nunca en el suelo el cual presenta más humedad en estas zonas. N. guanabacoae es la especie de Nops antillanos colectada en la mayor cantidad de hábitats (aunque pudiera tratarse de un grupo de especies afines). Puede vivir bajo piedra, troncos, plantas de Agave sp. de bosques secos, pinares, charrascales, pastizales y matorrales del archipiélago cubano. De las tres especies de La Hispaniola, sólo N. ernestoi Sánchez-Ruiz, 2005 y N. gertschi Chickering, 1967 son endémicas de la isla. N. ernestoi se conoce de una sola localidad en la provincia La Vega en República Dominicana, mientras que N. gertschi y N. blandus presentan una distribución más amplia. Aparentemente las poblaciones de estas dos especies en la isla están bien delimitadas geográficamente. N. blandus (Bryant, 1942) ocupando toda la parte llana central entre las paleoislas norte y sur, y $N$. gertschi Chickering, 1967, viviendo al oeste en el Valle Oriental del Cibao y toda la parte baja del sur de la Sierra de Bahoruco (Fig. 3).

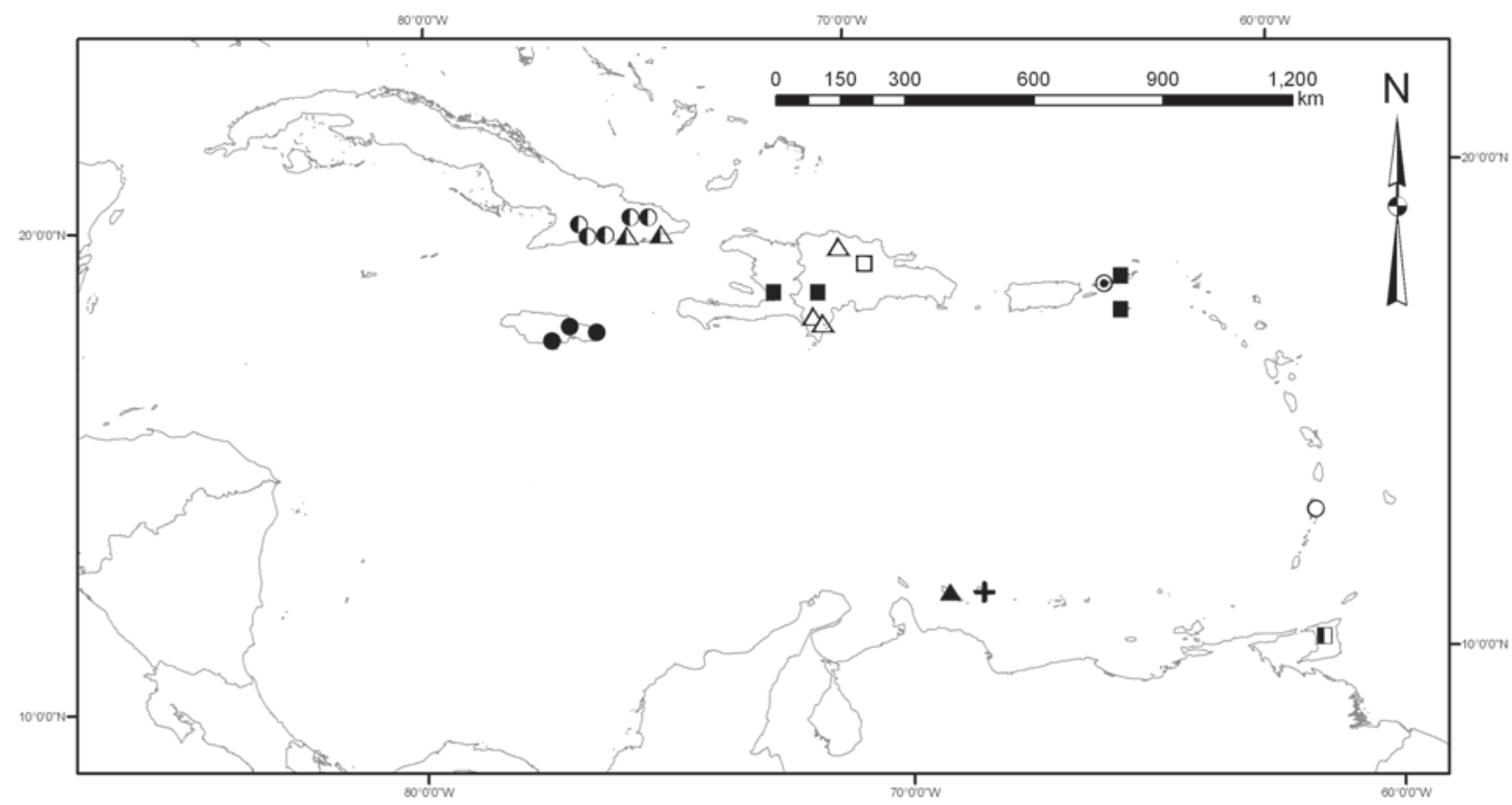

Figura 3. Distribución de $N$. blandus (cuadrado negro), N. coccineus (círculo negro), N. enae (círculo bicolor), N. ernestoi (cuadrado blanco), $N$. flutillus (triángulo negro), $N$. gertschi (triángulo blanco), N. glaucus (cruz negra), N. siboney (triángulo bicolor), N. toballus (círculo blanco), N. virginicus sp. nov. (círculo blanco y punto), N. craneae y N. simla (cuadrado bicolor). 
Atendiendo a la endemicidad de este género en Las Antillas, la nueva especie que se describe en este trabajo podría ser la única especie de Nops endémica de Islas Vírgenes, posiblemente microlocalizada y con poblaciones más pequeñas que Nops blandus, presente también en Islas Vírgenes y quien ha demostrado una mayor dispersión y adaptación a los hábitats. Trinidad es la única isla de las Antillas Menores donde viven simpátricamente dos especies de Caponiidae: $N$. simla Chickering, 1967, y N. craneae Chickering, 1967, ambas colectadas en el valle de Arima y sus alrededores. Hasta el momento no existen registros de Caponiidae en Puerto Rico. La falta de muestreos para localizar capónidos en Puerto Rico y gran parte de las Antillas Menores (Islas de Sotavento) dificulta los análisis biogeográficos. Sin embargo, teniendo en cuenta la distribución conocida de las especies, es muy probable que muchas de estas islas estén representadas por especies endémicas aún por describir.

\section{AGRADECIMIENTOS}

Este trabajo fue realizado gracias al apoyo financiero del Programa para estudio de colecciones del Museo Americano de Historia Natural (AMNH), la Sociedad Americana de Aracnología para Investigaciones Aracnológicas y BIOECO. Especial agradecimiento a Norman I. Platnick por el intercambio de especímenes y poner a disposición la colección de Caponiidae del AMNH y a Laura Leibensperger por facilitarnos el acceso a la colección del MCZ. A Martín Ramírez, Ernesto Ruiz, Ángela Rey y Otto Ríos por su colaboración, apoyo y preocupación. Especial agradecimiento además a Giraldo Alayón García por sus oportunos consejos, el préstamo de especímenes y la revisión crítica del manuscrito.

\section{LITERATURA CITADA}

Alayón García, G. 1976. Nueva especie de Nops MacLeay, 1839 (Araneae: Caponiidae) de Isla de Pinos, Cuba. Poeyana 148: 1-6.

Alayón García, G. 1977. Descripción del macho de Nops ludovicorum y redescripción de la hembra de Nops guanabacoae (Arachnida: Caponiidae). Poeyana 169: 1-8.

Archer, A. F. 1963. Arañas tejedoras de las Islas Vírgenes. Caribbean Journal Sciences. 3:207-208.

Bryant, E.1942. Notes on the spiders of the Virgin Islands. Bull. Mus. comp. Zool. Harv. 89: 317366.

Bryant, E.1948. The spiders of Hispaniola. Bull. Mus. comp. Zool. Harv. 100: 331-459.

Chickering, A. M. 1967a. The genus Nops (Araneae, Caponiidae) in Panama and the West Indies. Breviora Mus. Comp. Zool. 272: 19 pp.

Chickering, A. M. 1967b. Two new species of the genus Otiothops (Araneae, Palpimanidae) from the Virgin Islands. Psyche 74:203-207.

Chickering, A. M. 1968a. The genus Miagrammopes (Araneae, Uloboridae) in Panama and the West Indies. Breviora Mus. Comp. Zool. 289:1-28.

Chickering, A. M. 1968b. The genus Ischnothyreus (Araneae, Oonopidae) in Central America and the West Indies. Psyche 75:77-86.

Chickering, A. M. 1968c. The genus Scaphiella (Araneae, Oonopidae) in Central America and the West Indies. Psyche 75:135-156.

Chickering, A. M. 1969. The genus Stenoonops (Araneae, Oonopidae) in Panama and the West Indies. Breviora Mus. Comp. Zool. 339:1-35. 
Chickering, A. M. 1971. The genus Oonops (Araneae, Oonopidae) in Panama and the West Indies, Part 2. Psyche 78:203-214.

Chickering, A. M. 1973. Notes on Heteroonops and Triaeris (Araneae, Oonopidae). Psyche 80:227-229.

Exline, H., y H. W. Levi. 1962. American spiders of the genus Argyrodes (Araneae, Theridiidae). Bull. Mus. Comp. Zool. Harvard, 127:75-204.

Galiano, M. E. 1962. Redescripciones de especies del género Lyssomanes Hentz, 1845, basadas en los ejemplares típicos. Descripción de una especie nueva (Araneae, Salticidae). Acta Zool. Lilloana, 18: 45-97.

Hasselt, A. W. M. van. 1887. Études sur le genre Nops. Tijdschr. Ent. 30: 67-86.

Muchmore W. B. 1993. List of terrestrial invertebrates of St. John, U.S. Virgin Islands (exclusive of Acarina and Insects), with some records of freshwater species. Caribbean Journal of Science, 29, No 1-2:30-38.

Nentwig, W. 1993. Spiders of Panama: Biogeography, investigation, phenology, check list, key and bibliography of a tropical spider fauna. Sandhill Crane Press, Gainesville, Florida, 274 pp.

Petrunkevitch, A. 1926. Spiders from the Virgin Islands. Trans. Conn. Acad. Arts Sci. 28: 21-78.

Platnick, N. I. 1975. A revision of the palpimanid spiders of the new subfamily Otiothopinae (Araneae, Palpimanidae). Amer. Mus. Novitates 2562:1-32.

Platnick, N. I. 1978. A new Microsa from the Bahama Islands (Araneae, Gnaphosidae). J. Arachnology. 5:182-183.

Platnick, N. I., y M. V. Shadab.1976. A revision of the neotropical spider genus Zimiromus, with notes on Echemus (Araneae, Gnaphosidae). Amer. Mus. Novitates 2609:1-24.

Platnick, N. I., y M. V. Shadab. 1977. A new genus of the spider subfamily Gnaphosinae from the Virgin Islands (Araneae, Gnaphosidae). J. Arachnology. 3:191-194.

Platnick, N. I., y M. V. Shadab. 1979. A review of the spider genera Anapisona and Pseudanapis (Araneae, Anapidae). Amer. Mus. Novitates 2672:1-20.

Platnick, N. I., y M. V. Shadab. 1980. A revision of the spider genus Cesonia (Araneae, Gnaphosidae). Bull. Amer. Mus. Nat. Hist. 165:335-386.

Platnick, N. I., y M. V. Shadab.1982. A revision of the American spiders of the genus Camillina (Araneae, Gnaphosidae). Amer. Mus. Novitates 2748:1-38.

Platnick, N. I. y M. U. Shadab. 1989. A review of the spider genus Teminius (Araneae, Miturgidae). Amer. Mus. Novitates. 2963: 1-12.

Sánchez-Ruiz, A. 2004. Current taxonomic status of the family Caponiidae (Arachnida, Araneae) in Cuba with the description of two new species. Revista Ibérica de Aracnología 9: 95-102.

Sánchez-Ruiz, A. 2005a. Una nueva especie de Nops MacLeay, 1839 (Araneae, Caponiidae) de República Dominicana, Antillas Mayores. Revista Ibérica de Aracnología 11. 23-27. 
Sánchez-Ruiz, A. 2005b. Spiders. En: Fong, A., D. Maceira, W. S. Alverson y J. M. Shopland (Eds.). Cuba: Siboney-Juticí. Rapid Biological Inventories 10. The Field Museum. Chicago. EE.UU. 47-50.

Simon, E.1891. On the spiders of the island of St. Vincent. Part 1. Proc. zool. Soc. Lond. 1891: 549-575.

Simon, E.1892. Histoire naturelle des araignées. Paris, 1: 1-256.

Teruel, R. y A. Sánchez-Ruiz. 2000. Nota sobre la depredación de un escorpión (Scorpiones: Buthidae) sobre una araña (Araneae: Caponiidae). Biodiversidad de Cuba Oriental, 4: 82-83. 